\title{
Stability Analysis for Continuous T-S Fuzzy Models Having Uncertainties: A Systematic Approach
}

\author{
Mohammad Shekaramiz ${ }^{1, *}$ and Farid Sheikholeslam ${ }^{2}$ \\ 1,* ECE Dept., Utah State University, Utah, U.S.A. \\ ${ }^{2}$ ECE Dept., Isfahan University of Technology, Isfahan, Iran
}

\begin{abstract}
Stability analysis of continuous-time unforced T-S fuzzy systems is considered. Based on the pairwise commutative feature that usually occurs among the state matrices in switching systems, here we reformularize an existing discrete-time stability analysis approach to its continuous-time domain version. Using a common quadratic Lyapunov function, we first present a systematic approach for the asymptotic stability of such systems in case where the state matrices of sub-systems follow pairwise commutative feature. We then show that the method is not only limited to systems following such feature but rather can be applied to wider category. Finally, we investigate the maximum permissible uncertainty bound for holding the stability when the uncertainties in the system belong to convex sets.
\end{abstract}

Keywords : Stability, Takagi-Sugeno (T-S) fuzzy systems, Pairwise commutative, Maximum uncertainty bound.

\section{Introduction}

Stability is one of the most important issues in any control system. Since fuzzy systems have been proved to be applicable to many industrial applications, analyzing the stability of such systems is of great importance. However, fuzzy systems are essentially nonlinear and stability analysis of such systems is difficult. In order to tackle this problem, Takagi and Sugeno [1] proposed Takagi-Sugeno (T-S) fuzzy model, where the dynamic model is composed of a set of local linear sub-systems via the fuzzy inference. Based on the Lyapunov direct method, Tanaka and Sugeno [2] showed that the stability of a T-S fuzzy model can be guaranteed via finding a common symmetric positive-definite matrix $\mathrm{P}$ for all the subsystems. An unforced continuous-time T-S fuzzy model can be represented as

Rule $:$ if $x_{1}(t)$ is $M_{1}^{i}$ and $\ldots x_{n}(t)$ is $M_{n}^{i}$ then $\dot{X}(t)=A_{i} X(t), i=1,2, \ldots, n$.

where, $X(t)$ is the state vector, $\mathrm{n}$ is the number of IF-THEN rules, Rule is the $\mathrm{i}$-th fuzzy inference rule and $M_{j}^{i}$ is the fuzzy set. The global T-S fuzzy system is then inferred as 


$$
\dot{X}(t)=\sum_{i=1}^{n} h_{i}(X(t)) A_{i} X(t), \quad h_{i}(x(t))=\frac{\omega_{i}(x(t))}{\sum_{i=1}^{n} \omega_{i}(x(t))},
$$

where, $\omega_{i}(x(t))=\prod_{j=1}^{n} M_{j}^{i}\left(x_{j}(t)\right)$ is the firing strength of the $\mathrm{i}$-th rule and $M_{j}^{i}\left(x_{j}(t)\right)$ is the membership grade of $x_{j}(t)$ to the fuzzy set $M_{j}^{i}$.

Tanaka and Sugeno presented the following theorem for verifying the stability of system (2).

Theorem 1 [2]: The continuous fuzzy system (2) is globally asymptotically stable if there exists a common symmetric positive-definite matrix $\mathrm{P}$ such that

$$
A_{i}^{T} P+P A_{i}\langle 0, i=1,2, \ldots, n \text {. }
$$

The set of inequalities in (3) can also be rewritten as $A_{i}^{T} P+P A_{i}=-Q_{i}, Q_{i}=Q_{i}^{T}>0, i=1,2, \ldots, n$.

It can be seen from (3) that matrix $P$ must satisfy $n$ Lyapunov inequalities. But in many cases, especially by increasing the number of fuzzy rules, finding such matrix that satisfies set of Lyapunov's inequalities seems to be hard or a futile attempt. During the past decades, many researchers attempted to reduce the conservatism of Tanaka and Sugeno's model [3-10]. For example, Tanaka et al. [5] studied the stability problem by taking into account the time derivative property of membership functions. They defined a fuzzy Lyapunov function by fuzzily blending quadratic Lyapunov functions. Shekaramiz and Sheikholeslam [10] represented a method for checking the stability of such systems based on the properties in estimating the spectra of Hermitian matrices [13].

One of the first systematic methods for obtaining the common matrix $\mathrm{P}$ was proposed by Narendra et al. [11] for the stability of switching systems having pairwise commutative state matrices. Based on [11], Joongseon Joh et al. [12] introduced a novel systematic approach for finding a common positive-definite matrix $\mathrm{P}$ for discrete T-S models in case of having pairwise commutative state matrices. They also obtained criteria under which even if the state matrices are not pairwise commutative, a common matrix P can still be found. Thanks to their conducive work, it is possible to extend such approach to the continuous T-S models. Such possibility for continuous-time T-S models is what we seek in this paper. Here, we reformuralize the systematic method proposed by Joh et al. [12] for the stability analysis of continuous-time T-S fuzzy models. In this case, we show that even if the state matrices are not pairwise commutative, when additional condition holds, we can still check the stability 
via the proposed systematic method. This implies that the algorithm is not limited to systems having pairwise commutative state matrices but rather such property can be relaxed in some problems. The problem is then extended to fuzzy systems having uncertainties belong to convex sets. We find the maximum permissible uncertainty bounds under which the quadratic stability of the system is held.

\section{Proposed Stability Approach for Continuous-Time T-S Systems}

In this section, we provide three corollaries for the stability anlalysis of T-S continuoustime fuzzy models. These are essentially the reformuralized version of their corresponding discrete-time domain proposed in [12]. Below, we first provide a systematic approach for continuous-time T-S systems having stable and pariwise commutative state matrices.

Corollary 1: Assume that all the state matrices $A_{i}$ in the T-S model (1) are asymptotically stable and follow the pairwise commutative propoery i.e., $A_{j} A_{j+1}=A_{j+1} A_{j}, j=1,2, \ldots, n-1$. Consider the following Lyapunov equations

$A_{1}^{T} P_{1}+P_{1} A_{1}=-Q, A_{2}^{T} P_{2}+P_{2} A_{2}=-P_{1}, \ldots, A_{n}^{T} P_{n}+P_{n} A_{n}=-P_{n-1}$

where $Q>0$ and $P_{i}, i=1,2, \ldots, n$. is the unique positive definite symmetric solution of the $i$ th equation. Then the following LMIs are always held

$A_{i}^{T} P_{n}+P_{n} A_{i}\langle 0 \quad, i=1,2, \ldots, n$.

This means that for any unforced continuous-time T-S model having asymptotically stable and pairwise commutative state matrices, there always exist a common positive-definite matrix $P_{n}$ that satisfies the set of inequalities (5). In other words, such systems are globally asymptotically stable.

Proof: The proof is omited. More details can be found in [16].

Though the pairwise commutative feature rarely exists in continuous-time T-S systems, it is still possible to apply the systemic approach in corollary 1 to a wider category. Below, we show that the described systematic approach can also be applied for the stability analysis of continuous-time T-S models where the nominal state matrices are stable and pairwise commutative but accompanied with noise or perturbations. Suppose that T-S system (1) has 
nominal pairwise commutative and asymptotically stable state matrices $\tilde{A}_{i}$ accompanied with perturbations $\Delta A_{i}$ in the following form

$A_{i}=\tilde{A}_{i}+\Delta A_{i}, i=1,2, \ldots, n$

where $A_{i}$ matrices do not follow the pairwise commutative feature anymore. Gu et al. [14-15] provided an approach for the linear systems with uncertainty for the case where uncertainty belongs a convex set. Joh et al. [12] used of such assumption and provided a criterion which sought the stability of discrete-time T-S fuzzy models. Based on such approach, here we provide a stability analysis approach that can be applied to continuous-time T-S systems.

Corollary 2: Consider the following fuzzy model.

Rule $^{i}$ : if $x_{1}(t)$ is $M_{1}^{i}$ and $\ldots x_{n}(t)$ is $M_{n}^{i}$ then $\dot{X}(t)=\left[A_{i}+\Delta A_{i}(t)\right] X(t), i=1,2, \ldots, n$.

where, $A_{i}$ are the nominal state matrices of sub-systems and are assumed to be asymptotically stable and pairwise commutative. Matrices $\Delta A_{i}(t)$ are time varying uncertainties with $\Delta A_{i}(t) \in \Omega_{i}$ and $\Omega_{i}$ are compact and convex sets. Then, the uncertain system (7) is quadratically stable if the following set of inequalities is held for vertices of uncertainty sets.

$$
\max _{E i_{I} \in \Omega i_{I}} \lambda_{\max }\left[\left(A_{i}+E_{i}\right)^{T} P_{n}+P_{n}\left(A_{i}+E_{i}\right)\right]<0, \quad i=1,2, \ldots, n .
$$

where, $P_{n}$ is a fixed symmetric positive-definite matrix obtained from applying the systematic approach of corollary 1 to the nominal state matrices $A_{i}$.

Proof: Refer to $[12,16]$.

Corollary 3: Consider the continuous-time uncertain T-S fuzzy model (7). The nominal state matrices $A_{i}$ are asymptotically stable and pairwise commutative, and $\Delta A_{i}(t) \in \Omega_{\delta_{i}}$. Assume that the maximum permissible values for the sets $\Omega_{1}, \Omega_{2}, \ldots, \Omega_{n}$ be in the form below.

$$
\Lambda_{i}=\left\{\left(\delta_{i}\right)_{\max } E_{\text {fixed }_{i}} \mid E_{\text {fixed }_{i}} \in \Omega_{\text {fixed }_{i}}\right\}, i=1,2, \ldots, n \text {. }
$$

Then,

$\frac{1}{\left(\delta_{i}\right)_{\max }}=\max _{E_{\text {frxed }_{i} \in \Omega_{\text {fixed }}}} \frac{1}{\delta_{i}^{*}\left(E_{\text {fixed }_{i}}\right)}, i=1,2, \ldots, n$.

and, 


$$
\frac{1}{\delta_{i}^{*}\left(E_{\text {fixed }_{i}}\right)}=\max \left\{\frac{1}{\delta_{i}} \mid \operatorname{det}\left[\left(\frac{1}{\delta_{i}} A_{i}+E_{\text {fixed }_{i}}\right)^{T} \quad P_{n}\left(\frac{1}{\delta_{i}} A_{i}+E_{\text {fixed }_{i}}\right)\right]=0\right\} .
$$

Therefore, the system (7) with the defined state matrices is quadratically stability for all $\delta_{i}>\left(\delta_{i}\right)_{\max }$.

Remark: We considered two set of stability problems. First, we assumed that the state matrices in (1) are stable and pairwise commutative. The second case assumed the same properties on the nominal state matrices but in case of having uncertainties in the system. For the stability analysis of both cases a systematic approach was proposed. Notice that the second problem can be infered as a continuous-time T-S system in which the state matrices do not follow the pairwise commutativity. This means that one can divide each original state matrix of the system into two parts i.e., the nominal and the remained portion, where the nominal parts satisfy the pairwise commutative property and the remained portions are considered as uncertainties. Then, if the remained portions belong to convex sets, it is straight forward to apply the above results. Therefore, the proposed approach is not limited only to those continuous-time T-S models that satisfy the pairwise commutativity. We will show an example for this case in example 3 in the next section.

\section{Numerical Results}

Example 1: Consider the T-S system of (7) with the following state matrices.

$$
A_{1}=\left[\begin{array}{cc}
-3 & 7 \\
2 & -9
\end{array}\right], A_{2}=\left[\begin{array}{cc}
-4 & 14 \\
4 & -16
\end{array}\right], A_{3}=\left[\begin{array}{cc}
-1.6 & 2.8 \\
0.8 & -4
\end{array}\right]
$$

All the above state matrices are both asymptotically stable and pairwise commutative. Now, consider the same uncertainty sets as defined in $[12,15]$ i.e.,

$$
\Delta A_{1}(t)=\Delta A_{2}(t)=\Delta A_{3}(t)=\left[\begin{array}{ll}
d_{1}(t) & d_{2}(t) \\
d_{2}(t) & d_{1}(t)
\end{array}\right]
$$

where, $d_{2}(t), d_{1}(t)$ are uncertainty parameters defined by $\left|d_{1}(t)\right| \leq \rho,\left|d_{2}(t)\right| \leq 2 \rho$, and $\rho=0.1$. Such uncertainty sets $\Omega_{1}, \Omega_{2}, \Omega_{3}$ can be written in the following form.

$$
\Omega_{1}=\Omega_{2}=\Omega_{3}=\left[\begin{array}{ll}
\left\{d_{1}|| d_{1} \mid \leq r\right\} & \left\{d_{2}|| d_{2} \mid \leq 2 r\right\} \\
\left\{d_{2}|| d_{2} \mid \leq 2 r\right\} & \left\{d_{1}|| d_{1} \mid \leq r\right\}
\end{array}\right]
$$

It is obvious that the sets $\Omega_{1}, \Omega_{2}, \Omega_{3}$ are convex and there exists four vertices for each set. 
Such points are

$$
E_{i}^{1}=\left[\begin{array}{cc}
-\rho & -2 \rho \\
-2 \rho & -\rho
\end{array}\right], E_{i}^{2}=\left[\begin{array}{cc}
-\rho & 2 \rho \\
2 \rho & -\rho
\end{array}\right], E_{i}^{3}=\left[\begin{array}{cc}
\rho & -2 \rho \\
-2 \rho & \rho
\end{array}\right], E_{i}^{4}=\left[\begin{array}{cc}
\rho & 2 \rho \\
2 \rho & \rho
\end{array}\right], i=1,2,3 .
$$

Using corrallary 1 , it is straight forward to find below matrix $P_{3}$ as a common positive definte for the nominal state matrices of all sub-systems

$$
P_{3}=\left[\begin{array}{cc}
0.2032 & 0.182 \\
0.182 & 0.1633
\end{array}\right],
$$

which verifies that the nominal system is stable. Below, we investigate the quadratic stability of the whole system.

Table 1: Investigating the protruded points of uncertainty for the sub-system 1.

\begin{tabular}{c|c|c}
$E_{i}$ & $\lambda_{1,2}\left[\left(A_{1}+E_{i}\right)^{T} P_{3}+P_{3}\left(A_{1}+E_{i}\right)\right]$ & $\lambda_{\max }\left[\left(A_{1}+E_{i}\right)^{T} P_{3}+P_{3}\left(A_{1}+E_{i}\right)\right]$ \\
\hline \hline$E_{i}^{1}$ & $-1.0981,-0.0034$ & -0.0034 \\
\hline$E_{i}^{2}$ & $-0.8069,-0.0034$ & -0.0034 \\
\hline$E_{i}^{3}$ & $-0.9516,-0.0033$ & -0.0033 \\
\hline$E_{i}^{4}$ & $-0.6604,-0.0033$ & -0.0033
\end{tabular}

Table 2: Investigating the protruded points of uncertainty for the sub-system 2.

\begin{tabular}{c|c|c}
$E_{i}$ & $\lambda_{1,2}\left[\left(A_{2}+E_{i}\right)^{T} P_{3}+P_{3}\left(A_{2}+E_{i}\right)\right]$ & $\lambda_{\max }\left[\left(A_{2}+E_{i}\right)^{T} P_{3}+P_{3}\left(A_{2}+E_{i}\right)\right]$ \\
\hline \hline$E_{i}^{1}$ & $-0.5119,-0.0062$ & -0.0062 \\
\hline$E_{i}^{2}$ & $-0.2213,-0.0056$ & -0.0056 \\
\hline$E_{i}^{3}$ & $-0.3654,-0.0061$ & -0.0061 \\
\hline$E_{i}^{4}$ & $-0.0762,-0.0041$ & -0.0041
\end{tabular}

Table 3: Investigating the protruded points of uncertainty for the sub-system 3.

\begin{tabular}{c|c|c}
$E_{i}$ & $\lambda_{1,2}\left[\left(A_{3}+E_{i}\right)^{T} P_{3}+P_{3}\left(A_{3}+E_{i}\right)\right]$ & $\lambda_{\max }\left[\left(A_{3}+E_{i}\right)^{T} P_{3}+P_{3}\left(A_{3}+E_{i}\right)\right]$ \\
\hline \hline$E_{i}^{1}$ & $-0.8637,-0.0014$ & -0.0014 \\
\hline$E_{i}^{2}$ & $-0.5725,-0.0015$ & -0.0015 \\
\hline$E_{i}^{3}$ & $-0.7172,-0.0013$ & -0.0013 \\
\hline$E_{i}^{4}$ & $-0.4260,-0.0014$ & -0.0014
\end{tabular}

Therefore, for any arbitraty uncertainties belonging to the defined uncertainty sets $\Omega_{1}, \Omega_{2}, \Omega_{3}$, the system is quadratically stable.

Example 2: Consider the T-S fuzzy model of Ex. 1. Assume that $E_{\text {fixed }_{i}}^{1}=\left[\begin{array}{ll}-1 & -2 \\ -2 & -1\end{array}\right], E_{\text {fixed }_{i}}^{2}=\left[\begin{array}{cc}-1 & 2 \\ 2 & -1\end{array}\right], E_{\text {fixed }_{i}}^{3}=\left[\begin{array}{cc}1 & -2 \\ -2 & 1\end{array}\right], E_{\text {fixed }_{i}}^{4}=\left[\begin{array}{ll}1 & 2 \\ 2 & 1\end{array}\right], i=1,2,3 . \quad \Omega_{\text {fixed }_{i}}=\left\{E_{\text {fixed }_{i}}^{1}, E_{\text {fixed }_{i}}^{2}, E_{\text {fixed }_{i}}^{3}, E\right.$ 
The goal is to find the maximum uncertainty bound under which the stability is guaranteed. Using corollary 1 and 2, the results are shown in the following tables.

Table 4: Applying (10) to calculate $\delta_{i, \max }$.

\begin{tabular}{c|c}
$A_{i}$ & $\delta_{i, \max }$ \\
\hline \hline$A_{1}$ & $\delta_{1, \max }=0.2898$ \\
\hline \multirow{2}{*}{$A_{2}$} & $\delta_{2, \max }=$ \\
& 0.11675 \\
\hline \multirow{2}{*}{$A_{3}$} & $\delta_{3, \max }=$ \\
& 0.19117
\end{tabular}

Table 5: Applying (11) to calculate $\delta_{i}^{*}$.

\begin{tabular}{l|c|c|c} 
& $A_{1}$ & $A_{2}$ & $A_{3}$ \\
\hline$E_{\text {fixed }_{i}}^{j}$ & $\delta_{1}^{*}$ & $\delta_{2}^{*}$ & $\delta_{3}^{*}$ \\
\hline \hline$E_{\text {fixed }_{i}}^{1}$ & 1.10249 & 1.6434 & 0.53599 \\
\hline$E_{\text {fixed }_{i}}^{2}$ & 0.4658 & 0.24274 & 0.28775 \\
\hline$E_{\text {fixed }_{i}}^{3}$ & 0.68599 & 0.78395 & 0.356095 \\
\hline$E_{\text {fixed }_{i}}^{4}$ & 0.2898 & 0.11675 & 0.19117
\end{tabular}

Tab.4 shows the maximum amount of the uncertainty bound corresponding to each rule, while maintaining the quadratic stability. Referring to Ex. 1, it is clear that the obtained $\rho=0.1$ is less than $\delta_{i, \max }, i=1,2,3$. This proves the quadratic stability of our system.

Example 3: Consider the below T-S fuzzy model borrowed from [7].

$R^{1}:$ if $x_{1}$ is $N$ and $x_{2}$ is $N$, then $\quad \dot{X}(t)=\left[\begin{array}{cc}-6.4 & 2 \\ 2 & -5\end{array}\right] X(t)$

$R^{2}:$ if $x_{1}$ is $Z$ and $x_{2}$ is $N$, then $\dot{X}(t)=\left[\begin{array}{cc}-5.2 & 2 \\ 2 & -4.3\end{array}\right] X(t)$

$R^{3}:$ if $x_{1}$ is $P$ and $x_{2}$ is $N$, then $\quad \dot{X}(t)=\left[\begin{array}{cc}-7 & 2 \\ 1.8 & -4\end{array}\right] X(t)$

$R^{4}:$ if $x_{1}$ is $N$ and $x_{2}$ is $Z$, then $\quad \dot{X}(t)=\left[\begin{array}{cc}-6 & 2 \\ 2 & -5\end{array}\right] X(t)$

$R^{5}$ : if $x_{1}$ is $Z$ and $x_{2}$ is $Z$, then $\quad \dot{X}(t)=\left[\begin{array}{cc}-7 & 1.2 \\ 3 & -5.2\end{array}\right] X(t)$
$R^{6}:$ if $x_{1}$ is $P$ and $x_{2}$ is $Z$, then $\quad \dot{X}(t)=\left[\begin{array}{cc}-6.2 & 2 \\ 2 & -4.5\end{array}\right] X(t)$

$R^{7}:$ if $x_{1}$ is $N$ and $x_{2}$ is $P$, then $\quad \dot{X}(t)=\left[\begin{array}{cc}-6.3 & 2.3 \\ 2 & -5\end{array}\right] X(t)$

$R^{8}:$ if $x_{1}$ is $Z$ and $x_{2}$ is $P$, then $\quad \dot{X}(t)=\left[\begin{array}{cc}-6.4 & 2.1 \\ 3 & -5.2\end{array}\right] X(t)$

$R^{9}:$ if $x_{1}$ is $P$ and $x_{2}$ is $P$, then $\quad \dot{X}(t)=\left[\begin{array}{cc}-5.8 & 2.2 \\ 2 & -5\end{array}\right] X(t)$

We define the nominal state matrix of the entire sub-systems as the average of all state matrices in the system i.e.,

$A_{0}=\frac{1}{n} \sum_{i=1}^{n} A_{i}=\left[\begin{array}{cc}-6.26 & 1.98 \\ 2.2 & -4.8\end{array}\right]$

Then, we can change the sub-systems into $\dot{X}(t)=\left(A_{i}+\Delta A_{i}(t)\right) X(t), i=1,2, \ldots, 9$, where $\Delta A_{i}$ are defined as follows. 


$$
\begin{aligned}
& \Delta A_{1}=\left[\begin{array}{cc}
-0.14 & 0.2 \\
-0.2 & -0.2
\end{array}\right], \Delta A_{2}=\left[\begin{array}{cc}
0.26 & 0.2 \\
-0.2 & -0.2
\end{array}\right], \Delta A_{3}=\left[\begin{array}{cc}
-0.04 & 0.32 \\
-0.2 & -0.2
\end{array}\right], \Delta A_{4}=\left[\begin{array}{cc}
1.06 & 0.2 \\
-0.2 & 0.5
\end{array}\right], \Delta A_{5}=\left[\begin{array}{cc}
-0.74 & -0.78 \\
0.8 & -0.4
\end{array}\right], \\
& \Delta A_{6}=\left[\begin{array}{cc}
-0.14 & 0.12 \\
0.8 & -0.4
\end{array}\right], \Delta A_{7}=\left[\begin{array}{cc}
-0.74 & 0.2 \\
-0.4 & 0.8
\end{array}\right], \Delta A_{8}=\left[\begin{array}{cc}
0.06 & 0.2 \\
-0.2 & -0.3
\end{array}\right], \Delta A_{9}=\left[\begin{array}{cc}
0.46 & 0.22 \\
-0.2 & -0.2
\end{array}\right]
\end{aligned}
$$

Since all the sub-systems have the same nominal state matrix $A_{0}$, the pairwise commutative characteristic is already satisfied (trivial: $A_{0} A_{0}=A_{0} A_{0}$ ). Therefore, the common positive definite matrix $P_{n}$ for the system with nominal state matrices can be easily obtained from applying corollary 1 . As a result,

$$
A_{0}^{T} P_{n}+P_{n} A_{0}=-Q, Q=I \Rightarrow P_{n}=\left[\begin{array}{cc}
0.0943 & 0.04096 \\
.04096 & 0.1211
\end{array}\right] \text {. }
$$

Now, assume that the uncertainties region are defined as follows

$$
E_{\text {fixed }_{i}}^{1}=\left[\begin{array}{ll}
-1 & -2 \\
-2 & -1
\end{array}\right], E_{\text {fixed }_{i}}^{2}=\left[\begin{array}{cc}
-1 & 2 \\
2 & -1
\end{array}\right], E_{\text {fixed }_{i}}^{3}=\left[\begin{array}{cc}
1 & -2 \\
-2 & 1
\end{array}\right], E_{\text {fixed }_{i}}^{4}=\left[\begin{array}{ll}
1 & 2 \\
2 & 1
\end{array}\right], i=1,2, \ldots, 9
$$

It is obvious that all the actual uncertainties on the system, $\Delta \mathrm{A}_{i}$, lie within the above uncertainty region. Below, we seek the maximum permissible uncertainty bound in which the quadratic stability of the system is fulfilled.

Table 6: Applying (10) to calculate the values of $\delta_{i, \max }$.

\begin{tabular}{c|c|c|c|c}
$E_{\text {fixed }_{i}}^{j}$ & $E_{\text {fixed }_{i}}^{j}$ & $E_{\text {fixed }_{i}}^{j}$ & $E_{\text {fixed }_{i}}^{j}$ & $E_{\text {fixed }_{i}}^{j}$ \\
\hline \hline$\delta_{i, \text { max }}$ & 7.45643 & 3.3527 & 2.4921 & 1.1205
\end{tabular}

By defining $\rho=\min _{i} \delta_{i, \max }$ and the results in Tab.6, the maximum permissible of uncertainty bound becomes $\rho=1.1205>1$, which captures the uncertainties of the sub-systems. Hence, the obtained matrix $P_{n}$ is a common positive definite matrix $\mathrm{P}$ for the whole system and the system is stable. Table below verifies the stability.

Table 7: Investigating the maximum coefficient values of uncertainty.

\begin{tabular}{c|c|c|c|c|c|c|c|c|c}
$A_{i}$ & $A_{1}$ & $A_{2}$ & $A_{3}$ & $A_{4}$ & $A_{5}$ & $A_{6}$ & $A_{7}$ & $A_{8}$ & $A_{9}$ \\
\hline$\lambda_{1,2}\left[A_{i}^{T} P_{n}+P_{n} A_{i}\right]$ & -1.0790, & -0.9029, & -0.7883, & -1.0325, & -1.1670, & -1.0607, & -1.0196, & -1.1311, & -1.0313, \\
& -1.0577 & -0.7769 & -1.1746 & -0.9676 & -1.0688 & -1.0014 & -1.0273 & -0.9175 & -0.9295
\end{tabular}

\section{Conclusion}

Stability of continuous T-S fuzzy models was studied. First, we showed that if the state matrices are asymptotically stable and pairwise commutative, then the whole fuzzy system is 
asymptotically stable. Then, the stability problem of such systems with uncertainties was investigated. In this case, an approach was introduced to check the quadratic stability of such systems. Finally, under the assumption that uncertainties belong convex sets, we sought the maximum permissible bounds such that the quadratic stability of the system is held. We showed that the proposed approach is not limited to systems with pairwise commutative characteristic but rather can be applied to wider category.

\section{References}

[1] Takagi, T., Sugeno, M. (1985). Fuzzy identification of systems and its applications to modeling and control, IEEE Trans. Systems Man Cybernetics, vol. 15, pp. 116-132.

[2] Tanaka, K., Sugeno, M. (1992). Stability analysis and design of fuzzy control systems, Fuzzy Sets and Systems, vol. 2, pp. 135-156.

[3] Kawamoto, S., Tada, K., Ishigame, A., Taniguchi, T. (1992). An approach to stability analysis of second order fuzzy systems, Proc. of IEEE Int. Conf. on Fuzzy Syst., pp. 1427-1434.

[4] Sheikholeslam, F., Shekaramiz, M. (2011). Stability criterion of Takagi-Sugeno models, Eight Int. Conf. on Fuzzy Syst. and Knowledge Discovery (FSKD), Proc. of IEEE, pp. 262-267.

[5] Tanaka, K., Hori, T., Wang, H. O. (2001). A fuzzy Lyapunov approach to fuzzy control system design, Proc. of the American Control Conf., pp. 4790-4795, June.

[6] Wang, W. J., Sun, C. H. (2005). Relaxed stability and stabilization conditions for a T-S fuzzy discrete system, Fuzzy Sets and Systems, vol. 156, pp. 208-225, May.

[7] Luoh, L. (2002). New stability analysis of T-S fuzzy systems with robust approach, Mathematics and Computers in Simulation, vol. 59, pp. 335-340.

[8] Pang, C. T., Guu, S. M. (2003). Sufficient conditions for the stability of linear TakagiSugeno free fuzzy systems, IEEE Trans. Fuzzy Syst., vol. 11, no. 5, pp. 695-700, Oct.

[9] Wang, W. J., Sun, C. H. (2004). A relaxed stability issues of linear Takagi- Sugeno fuzzy models, Proc. of the 2004 IEEE Int. Conf. on Networking, Sensing \& Control, pp. 21-23, March.

[10] Shekaramiz, M., Sheikholeslam, F. (2011). On the stability of continuous-time T-S, Eight Int. Conf. on Fuzzy Syst. and Knowledge Discovery (FSKD), Proc. of IEEE, pp. 226-230. 
[11] Narendra, K. S., Balakrishnan, J. (1994). A common Lyapunov function for stable LTI systems with commuting A-matrices, IEEE Trans. on Automatic Control, vol. 39, no. 12, pp. 2469-2471, Dec.

[12] Joh, J., Chen, Y. H., Langari, R. (1998). On the stability issues of linear Takagi-Sugeno fuzzy models, IEEE Trans. Fuzzy Syst., vol. 6, no. 3, pp. 402-410.

[13] Li, C. K., Li, R. C. (2005). A note on eigenvalues of perturbed Hermitian matrices, Linear Algebra and Its Applications, vol. 395, pp. 183-190.

[14] Gu, K., Zohdy, M., Loh, N. K. (1990). Necessary and sufficient conditions of quadratic stability of uncertain linear systems, IEEE Trans. on Automatic Control, vol. 35, pp. 601-604, May.

[15] Gu, K., Chai, W. J., Loh, N. K. (1990). Toward less conservative stability criterion for discrete-time linear uncertain systems, in Proc. Amer. Contr. Conf., San Diego, CA, pp. 1145-1149, June.

[16] Shekaramiz, M. (2006). Stability analysis of Takagi-Sugeno fuzzy systems, Master's thesis, Isfahan University of Technology, Isfahan, Iran. 